\section{CIRED 2003, Barcelona}

Die 17. Internationale Konferenz über Stromverteilung (CIRED) bewies neuerlich durch das breite internationale Angebot an technischen Themen und die hohe fachliche Kompetenz, mit der referiert und diskutiert wurde, dass sie die führende Veranstaltung zum Thema ist. Knapp 1000 Tagungsteilnehmer, um 230 mehr als 2001, hatten die theoretische Chance, über 600 Beiträge (um 300 mehr als 2001) zu diskutieren. Die Fülle des Angebots erforderte eine Straffung auf 470 Papiere und Aufteilung auf parallele Sitzungen. Während die sechs Sessionen siehe nachfolgende Berichte in dieser Ausgabe der e \& i - vor allem für die Aktualisierung der Papiere und Diskussion mit den Autoren diente, stand bei den Round Tabel-Gesprächen die breite Diskussion bestimmter Themen im Vordergrund.

Die Themenfelder reichten von der neuesten Entwicklung der klassischen Netzkomponenten über Fragen des Netzbetriebs, Netzschutz und Steuerung, die Versorgungsqualität bis zu Asset Management, Netzplanung, Instandsetzung und Umweltschutz. Aber auch die Deregulierung, Organisationsfragen sowie dezentrale Einlieferung standen auf der Tagesordnung. Das Tagungsprogramm wurde durch Tutorials und das Academic Village, Exkursionen und eine Fachausstellung sowie Ausflüge zu Kulturgütern für die Begeleitpersonen abgerundet. So mancher Vortrag fand seine vertiefende Fortsetzung am Messestand eines Lieferanten, und eine neue Geschäftsbeziehung war angebahnt worden.

Durch die Vielzahl an Teilnehmern aus den unterschiedlichsten Bereichen wie Elektroindustrie, Energieversorgungsunternehmen, Universitäten, Prüfanstalten, Ingenieurbüros, Regulatoren und Behörden konnten vielfältige Aspekte beleuchtet werden.

Aus österreichischer Sicht ist zu vermerken, dass sowohl die Anzahl der Beiträge (10) als auch die Teilnehmerzahl (31) sehr zufriedenstellend und damit unser Land gut vertreten war.

Es dürfen auch die schöne Umgebung und die Gastfreundschaft in Barcelona nicht vergessen werden, die eine hervorragende Voraussetzung für kollegiale Gespräche sowohl innerhalb der österreichischen Delegation als auch mit Vertretern anderer Länder war.

Mein Dank geht an die Autoren der Beiträge und an die Berichterstatter sowie an den Geschäftsführer des Österreichischen Nationalkomitees (ÖNK) der CIRED, Herrn Prok. Dipl.-Ing. Eduard Vierlinger, die großteils inre Freizeit für die CIRED-Arbeit geopfert haben.

Die eigene Erfahrung zeigt mir, dass sich die Mühe, schon alleine wegen der Kontakte innerhalb der CIRED, lohnt. Ich lade daher technisch Interessierte ein, bei CIRED mitzuarbeiten. Informationen über die verschiedenen Möglichkeiten sind bei den Vorstandsmitgliedern zu bekommen.

Über die Organisation von CIRED international ist zu berichten, dass im Directing Commitee die Änderung der Satzung Zusammenarbeit zwischen IEE-AIM und den NC-Vertretern -

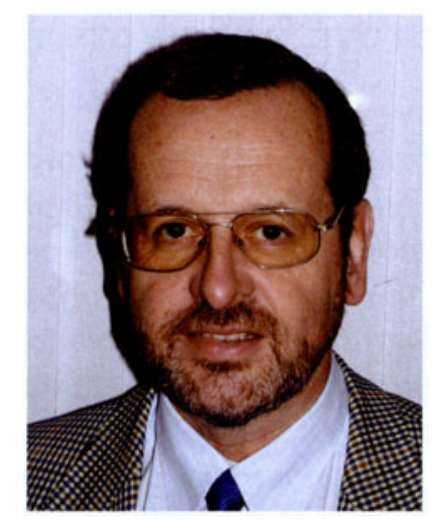

diskutiert wird, und dass bei der letzten Sitzung in Barcelona der Wechsel von Sander auf Haidvogl als österreichischen Vertreter erfolgte. Ein wichtiger Punkt ist die Aufnahme neuer Mitglieder, vor allem aus Staaten, die demnächst EU-Mitglied sein werden.

Die nächste CIRED-Konferenz wird von 6. bis 9. Juni 2005 in Turin, Italien, stattfinden, wozu ich schon jetzt recht herzlich einladen möchte

Zum Schluss noch ein Wort zum ÖNK der CIRED: Der langjährige Vorsitzende Direktor Prok. Dipl.-Ing. Dr. techn. Herbert Sander hat sich bereits in den beruflichen Ruhestand zurückgezogen und übergibt deshalb nach acht Jahren den Vorsitz an seinen Nachfolger. Dank seines unermüdlichen Einsatzes, nicht nur für die Belange der Stromversorgung in Österreich, sondern auch für eine positive Entwicklung der CIRED, konnte die Elektrizitätswirtschaft den ihr gebührenden Stellenwert in der Fachwelt und Gesellschaft erreichen.

Dank seines Weitblicks und der guten Zusammenarbeit mit dem Vorsitzenden von CIGRE-Österreich, Herrn Vorstandsdirektor Dr. Peter Morawek, und dem Geschäftsführer von CIRED und CIGRE, Herrn Prok. Dipl.-Ing. Eduard Vierlinger, ist es gelungen, die in Österreich relativ kleinen Gruppen CIRED und CIGRE derart zu koordinieren, dass Doppelgleisigkeiten künftig vermieden werden.

Sichtbares äußeres Zeichen dafür ist, dass heuer erstmals eine gemeinsame Veranstaltung - diesmal unter Vorsitz von CIRED - stattfand. Die Abstimmung der Satzungen und die personelle Verzahnung der beiden Komitees wird auch in Zukunft eine sehr enge und effiziente Zusammenarbeit ermöglichen.

Zum Dank für seinen Einsatz um die CIRED, bei dem auch der Humor niemals zu kurz kam, hat das ÖNK Herrn Direktor Dr. Sander Zum Ehrenmitglied ernannt und verbindet damit die besten Wünsche für den nächsten Lebensabschnitt!

\section{Dipl.-Ing. Herbert Haidvogl}

Vorsitzender des Österreichischen

Nationalkomitees der CIRED 